\title{
High glucose induces Drp1-mediated mitochondrial fission via the Orai1 calcium channel to participate in diabetic cardiomyocyte hypertrophy
}

\author{
Qing-Rui Wu (1) 1,2,3, Dan-Lin Zheng ${ }^{1,2,4}$, Pei-Ming Liu ${ }^{1,2,3}$, Hui Yang ${ }^{1,2}$, Lu-An Li $\mathbb{1}^{1,2,3}$, Su-Juan Kuang ${ }^{1,2}$, Ying-Yu Lai ${ }^{1,2,5}$,
} Fang Rao ${ }^{1,2}$, Yu-Mei Xue ${ }^{1,2}$, Ji-Jin Lin ${ }^{1,2}$, Shuang-Xin Liu' ${ }^{6}$, Chun-Bo Chen ${ }^{1,2,3,4}$ and Chun-Yu Deng $\mathbb{B}^{1,2,3,4,5}$

\section{Abstract}

Mitochondrial dysfunction and impaired $\mathrm{Ca}^{2+}$ handling are involved in the development of diabetic cardiomyopathy (DCM). Dynamic relative protein 1 (Drp1) regulates mitochondrial fission by changing its level of phosphorylation, and the Orai1 $\left(\mathrm{Ca}^{2+}\right.$ release-activated calcium channel protein 1) calcium channel is important for the increase in $\mathrm{Ca}^{2+}$ entry into cardiomyocytes. We aimed to explore the mechanism of Drp1 and Orai1 in cardiomyocyte hypertrophy caused by high glucose $(\mathrm{HG})$. We found that Zucker diabetic fat rats induced by administration of a high-fat diet develop cardiac hypertrophy and impaired cardiac function, accompanied by the activation of mitochondrial dynamics and calcium handling pathway-related proteins. Moreover, HG induces cardiomyocyte hypertrophy, accompanied by abnormal mitochondrial morphology and function, and increased Orai1-mediated $\mathrm{Ca}^{2+}$ influx. Mechanistically, the Drp1 inhibitor mitochondrial division inhibitor 1 (Mdivi-1) prevents cardiomyocyte hypertrophy induced by HG by reducing phosphorylation of Drp1 at serine 616 (S616) and increasing phosphorylation at 5637. Inhibition of Orai1 with single guide RNA (sgOrai1) or an inhibitor (BTP2) not only suppressed Drp1 activity and calmodulin-binding catalytic subunit A ( $\mathrm{CnA}$ ) and phosphorylated-extracellular signal-regulated kinase (p-ERK1/2) expression but also alleviated mitochondrial dysfunction and cardiomyocyte hypertrophy caused by HG. In addition, the CnA inhibitor cyclosporin A and p-ERK1/2 inhibitor U0126 improved HG-induced cardiomyocyte hypertrophy by promoting and inhibiting phosphorylation of Drp1 at S637 and S616, respectively. In summary, we identified Drp1 as a downstream target of Orai1-mediated $\mathrm{Ca}^{2+}$ entry, via activation by p-ERK1/2-mediated phosphorylation at $\mathrm{S} 616$ or CnA-mediated dephosphorylation at S637 in DCM. Thus, the Orai1-Drp1 axis is a novel target for treating DCM.
\end{abstract}

Correspondence: Chun-Bo Chen (gghccm@163.com) or

Chun-Yu Deng (chunyudeng@126.com)

'Guangdong Provincial Key Laboratory of Clinical Pharmacology, Research Center of Medical Sciences, Guangdong Provincial People's Hospital,

Guangdong Academy of Medical Sciences, 510080 Guangzhou, Guangdong, China

2Guangdong Cardiovascular Institute, Guangdong Provincial People's Hospital, Guangdong Academy of Medical Sciences, 510080 Guangzhou, Guangdong, China

Full list of author information is available at the end of the article These authors contributed equally: Qing-Rui Wu, Dan-Lin Zheng, Pei-Ming Liu Edited by A. Stephanou

\section{Introduction}

Diabetic cardiomyopathy (DCM), a major cardiovascular complication of diabetes mellitus (DM), is a myocardial dysfunction independent of coronary artery disease and hypertension ${ }^{1}$. DCM is characterized by left ventricular (LV) hypertrophy and cardiac dysfunction. Its pathogenesis is complex, and includes metabolic disorders, mitochondrial dysfunction, impaired cardiomyocyte calcium handling, and inflammation ${ }^{2}$. However, the correlation between calcium handling and mitochondrial dysfunction has not been reported in DCM. In-depth

\section{(c) The Author(s) 2021}

(c) (i) Open Access This article is licensed under a Creative Commons Attribution 4.0 International License, which permits use, sharing, adaptation, distribution and reproduction cc) in any medium or format, as long as you give appropriate credit to the original author(s) and the source, provide a link to the Creative Commons license, and indicate if changes were made. The images or other third party material in this article are included in the article's Creative Commons license, unless indicated otherwise in a credit line to the material. If material is not included in the article's Creative Commons license and your intended use is not permitted by statutory regulation or exceeds the permitted use, you will need to obtain permission directly from the copyright holder. To view a copy of this license, visit http://creativecommons.org/licenses/by/4.0/. 
study of the pathogenesis of DCM is helpful to discover new potential therapeutic targets. Therefore, this study aimed to explore the relationship between abnormal calcium regulation and mitochondrial dysfunction of DCM and the potential mechanism.

Mitochondrial homeostasis is essential for maintaining normal physiology in cardiomyocytes with high energy demands, but the underlying mechanism of aberrant mitochondrial morphology in DCM remains unclear. Abnormal mitochondrial morphology indicates an imbalance in mitochondrial dynamics, including mitochondrial fission and fusion. The main proteins that regulate changes in mitochondrial morphology are the dynamic-related GTPases, including dynamic-related protein 1 (Drp1), mitofusin (Mfn), and optic atrophy 1 (Opa1). Drp1 regulates mitochondrial fission, while fusion of the outer and inner mitochondrial membranes is regulated by $M f n 1 / 2$ and Opa1, respectively ${ }^{3}$.

Phosphorylation is a post-translational modification that plays an important role in cardiac hypertrophy ${ }^{4}$. Drp1 is mainly regulated by phosphorylation at serine 616 (S616) and S637. For example, mitochondrial fission is driven by extracellular signal-regulated kinase (ERK)mediated phosphorylation of Drp1 on S616, which plays a role in tumor proliferation ${ }^{5}$. In addition, calcineurin has been reported to induce S637 dephosphorylation, mediating $\mathrm{Ca}^{2+}$-induced Drp1-dependent mitochondrial fission $^{6}$. In summary, Drp1-dependent mitochondrial fission is mainly regulated by ERK-mediated phosphorylation at S616 or calcineurin-mediated dephosphorylation at S637. In addition, studies have shown that ERK can be activated by intracellular $\mathrm{Ca}^{2+}$ overload $^{7}$. The role of calcineurin as a $\mathrm{Ca}^{2+} /$ calmodulin-dependent serine/threonine phosphatase in cardiac hypertrophy and remodeling has been extensively investigated ${ }^{8}$. Calcineurin is structurally composed of two subunits': calmodulin-binding catalytic subunit $\mathrm{A}(\mathrm{CnA})$ and $\mathrm{Ca}^{2+}$-binding regulatory subunit $\mathrm{B}$. This study investigated the role of the calcium-regulated phosphatase CnA and kinase ERK in regulating Drp1 phosphorylation in diabetic cardiomyocytes. We sought to investigate whether $\mathrm{Ca}^{2+}$ disorders regulate Drp1 phosphorylation through the activation of ERK or CnA.

Dysregulation of $\mathrm{Ca}^{2+}$ handling is observed in DCM. The main mechanism of intracellular calcium signaling is store-operated $\mathrm{Ca}^{2+}$ entry (SOCE). The basic unit of SOCE is the accumulation of stromal interaction molecule 1 and $\mathrm{Ca}^{2+}$ release-activated calcium channel protein 1 (Orai1) at the endoplasmic reticulum (ER)-plasma membrane junction ${ }^{10}$. Expression of Orai1 protein was first detected in neonate mice heart, but Orai1 expression is more abundant in human myocardial tissue. Besides, the Orail channel is important for almost every cell type. In cardiomyocytes, the functions of Orail include its mediated SOCE in regulating ER $\mathrm{Ca}^{2+}$ content, diastolic
$\mathrm{Ca}^{2+}$, and cell growth during heart development, but more studies are needed to verify the role of Orai1mediated $\mathrm{Ca}^{2+}$ entry in the adult heart ${ }^{11}$.

Although recent studies have shown that Orail expression is upregulated in cardiac hypertrophy associated with MEK/ERK activation ${ }^{12}$, the role of Orai1-mediated SOCE in diabetes-induced cardiomyopathy is unclear. We hypothesized that Orai1-mediated SOCE promotes cardiac hypertrophy induced by high glucose (HG) via regulation of Drp1 phosphorylation. In the present study, we found that Drp1 and $\mathrm{Ca}^{2+}$ signaling pathway-related proteins were activated in hypertrophic rat hearts and in the DCM cell model. Moreover, inhibition of Drp1, Orai1, p-ERK1/2, and CnA prevents cardiomyocyte hypertrophy induced by HG by decreasing mitochondrial fission. These data provide evidence that the Orai1-Drp1 pathway may be a target for treating DCM.

\section{Materials and methods \\ Construction of animal models}

Ten male Zucker diabetic fat (ZDF) rats and ten male Zucker lean (ZL) rats were obtained from Beijing Vital River Laboratory Animal Technology Co. Ltd (China) at an age of 7 weeks. Six (six-weeks-old) male $\mathrm{db} / \mathrm{m}$ and $\mathrm{db} /$ $\mathrm{db}$ mice were purchased from GemPharmatech Co., Ltd (Nanjing, China). The animals were raised alone in specific pathogen-free conditions, with a $12 \mathrm{~h}$ light/dark cycle. After 1 week of adaptation, the rats were divided into two groups: $\mathrm{ZL}$, which received a normal diet and ZDF, which received a high-fat diet (Purina 5008). At 7 weeks, six $\mathrm{db} / \mathrm{db}$ male mice were randomly selected for intraperitoneal injection of Mdivi-1 (10 mg/kg, twice per week, 8 weeks, S716201, Selleck.cn, USA). The animals were considered to have type 2 diabetes at blood glucose concentration $\geq 11.1 \mathrm{mmol} / \mathrm{l}$. At $18-23$ weeks, changes in cardiac function in the two groups of rats were detected by Doppler echocardiography. Briefly, following anesthetization of the rats with $3 \%$ isoflurane, chest echocardiography was performed using a Visualsonics Vevo 2100 (Visualsonics Inc., Canada) ultrasound system with a 21$\mathrm{MHz}$ transducer. The recorded parameters were as follows: LV end-diastolic anterior wall thickness, LV wall end diastole, LV ejection fraction, and LV fraction shortening. Then, the body weight $(\mathrm{g})$, heart weight (HW; $\mathrm{mg})$, and $\mathrm{HW} /$ tibia length $(\mathrm{mg} / \mathrm{mm})$ were measured followed by anesthetization and sacrifice, and LV tissue was excised for analysis. This study was approved by the Ethics Committee and the Teaching and Research Committee (No. GDREC201208A).

\section{Electron microscopy}

The morphology of mouse ventricular mitochondria was observed by electron microscope. In brief, hearts excised from mice were immediately fixed in $2.5 \%$ 
glutaraldehyde (G1102, Servicebio, China). Then, ultrathin sections were examined with a JEM-1400plus transmission electron microscope (Japan Electron Optics Laboratory Co., Ltd, Tokyo). Mitochondrial area and aspect ratio (the ratio of length/width) were quantified by ImageJ. At least 100 randomly selected mitochondria were analyzed for each group.

\section{Cell culture, infection, and treatment}

Neonatal Sprague-Dawley rats (age, 1-3 days) were purchased from The Experimental Animal Center of Southern Medical University (Guangzhou, China). The hearts were removed, cut into pieces, and digested with $0.08 \%$ trypsin (T4799, Sigma, USA) and collagenaseII (LS004176, Worthington, USA). The purified cardiomyocytes were obtained by different adherence times, and $10 \mu \mathrm{M}$ bromodeoxyuridine (S7918, Selleck.cn, USA) was added to inhibit fibroblast proliferation. Neonatal rat cardiomyocytes (NRCMs) were cultured in Dulbecco's modified Eagle's medium (DMEM, C11885500BT, Gibco; Thermo Fisher Scientific, USA) supplemented with $10 \%$ fetal bovine serum (10099-414, Gibco) and $1 \%$ penicillin and streptomycin (15140122, Gibco) at $37^{\circ} \mathrm{C}$ and $5 \% \mathrm{CO}_{2}$. NRCMs were treated with $5.5 \mathrm{mM}$ glucose (normal glucose group), $33 \mathrm{mM}$ mannitol (high mannitol $[\mathrm{HM}]$ group), $33 \mathrm{mM}$ glucose (HG group), $10 \mu \mathrm{M}$ Mdivi-1/BTP2/U0126/cyclosporin A (Mdivi-1/BTP2/U0126/ CsA group), or HG and $10 \mu \mathrm{M}$ Mdivi-1/BTP2/U0126/CsA (HG + Mdivi-1/BTP2/U0126/CsA group). RNA interference was performed with single guide RNA (sgRNA). sgRNAs were introduced by lentiviral vector (genechem.com) according to the manufacturer's protocol. Mannitol, D-glucose, BTP2, CsA, U0126, and Mdivi-1 were obtained from Sigma.

\section{Immunofluorescence confocal microscopy Measurement of NRVM size}

NRVMs were fixed with $4 \%$ formaldehyde for $15 \mathrm{~min}$ at $37^{\circ} \mathrm{C}$ and washed three times with phosphate-buffered saline. Wheat germ agglutinin (WGA) Texas Red®-X conjugate (W21405, Gibco) was added and the cells were incubated for $10 \mathrm{~min}$ at room temperature. After removing the labeling solution and washing cells twice in phosphate-buffered saline, a laser copolymer microscope (SP5-FCS, Leica, Germany) was used to acquire images of labeled cells. NRVM areas were analyzed using the ImageJ software.

\section{Mitochondrial morphological measurement}

Two hundred nanomoles of Mito-Tracker Red CMXRos (C1049, Beyotime, China) was added and cultures were incubated $10 \mathrm{~min}$ at $37^{\circ} \mathrm{C}$ according to the instructions. A laser copolymer microscope was used to acquire fluorescence micrographs of labeled mitochondria. MiNA (Mitochondrial Network Analysis), which consists of a set of ImageJ macros, was used to measure mitochondrial morphological parameters from the confocal images ${ }^{13}$.

\section{Measurement of mitochondrial fission}

Mitochondrial were visualized in NRCMs by staining with $200 \mathrm{nM}$ Mito-Tracker Red CMXRos. Cells with fragmented mitochondria were identified as exhibiting mitochondrial fission.

\section{Mitochondrial membrane potential (MMP)}

MMP detection reagent JC-1 (C2006, Beyotime) was added and cultures were incubated $15 \mathrm{~min}$ at $37^{\circ} \mathrm{C}$ according to the manufacturer's instructions. Red and green fluorescence detected by laser confocal microscopy were from JC-1 aggregates and JC-1 monomers, respectively. ImageJ software was used to conduct semiquantitative analysis of the fluorescence intensity of MMP, and the ratio of red and green fluorescence was measured as the raw data of MMP.

\section{Intracellular $\mathrm{Ca}^{2+}$ measurement}

NRCMs were cultured in DMEM medium with Fluo4/ AM (F14201, Invitrogen, USA) at $5 \mu \mathrm{M}$ for $30 \mathrm{~min}$ at $37^{\circ} \mathrm{C}$. NRCMs were washed with $\mathrm{Ca}^{2+}$-free Tyrode's solution $(132 \mathrm{mM} \mathrm{NaCl}, 4.8 \mathrm{mM} \mathrm{KCl}, 1.2 \mathrm{mM} \mathrm{MgCl}$, $5 \mathrm{mM}$ glucose, $10 \mathrm{mM}$ HEPES, and $1.8 \mathrm{mM} \mathrm{CaCl}_{2}, \mathrm{pH}$ 7.4). Then, $1 \mu \mathrm{M}$ nifedipine was added to $\mathrm{Ca}^{2+}$-free Tyrode's solution and incubated for $2 \mathrm{~min}$ to inhibit L-type $\mathrm{Ca}^{2+}$ channel before the addition of thapsigargin $(2 \mu \mathrm{M})$ to induce $\mathrm{ER} \mathrm{Ca}^{2+}$ store depletion. At $12 \mathrm{~min}, \mathrm{Ca}^{2+}$ $\left(\mathrm{CaCl}_{2}, 2 \mathrm{mM}\right)$ was added to record the intracellular $\mathrm{Ca}^{2+}$ influx mediated by the SOC channel. Fluo4 fluorescence emission was monitored at $525 \mathrm{~nm}$ using a confocal laser scanning microscope (SP5-FCS, Leica).

\section{Total ATP determination}

Total ATP was determined using the ATP Assay Kit (S0026, Beyotime, China) according to the manufacturer's instructions. Briefly, ventricular tissue of mice $(\sim 20 \mathrm{mg})$ was homogenized with $100 \mu \mathrm{l}$ lysate and centrifuged at $12,000 \times g$ at $4{ }^{\circ} \mathrm{C}$ for $5 \mathrm{~min}$. The supernatant was mixed with the test solution and the relative light unit was measured by GloMax 20/20 (Promega, USA), and the results were compared to standards. Finally, the concentration of ATP was converted to $\mathrm{nmol} / \mathrm{mg}$ protein.

\section{Western blotting}

Total protein of ventricular tissues and NRCMs were extracted using RIPA Lysis Buffer (20-188, Millipore, USA) with Protease/Phosphatase Inhibitor Cocktail (5872, CST, USA). The protein concentration of the sample was determined by BCA Protein Assay Kit (P0011, Beyotime), quantified to $20 \mu \mathrm{g}$, separated by $10 \%$ sodium dodecyl 
sulfate-polyacrylamide gel electrophoresis, and transferred onto a PVDF membrane (IPVH00010, Millipore). The membrane was sealed with $5 \%$ skim milk (232100, BD Biosciences, USA) for $1 \mathrm{~h}$, and probed overnight at $4{ }^{\circ} \mathrm{C}$ with primary antibodies against different antigens, including $\beta$-myosin heavy chain ( $\beta$-MHC) (ARP41380, Aviva Systems Biology, USA), atrial natriuretic peptide (ANP) (ab180649, Abcam, USA), Orai1 (ab59330, Abcam), p-ERK1/2 (4370, CST), ERK1/2 (5013, CST), CnA (2614, CST), Drp1 (14647, CST), p-Drp1 ${ }^{\text {S616 }}$ (4494, CST), p-Drp1 ${ }^{\text {S637 }}$ (6319, CST), Mfn2 (9482, CST), and Opa1 (80471, CST). Protein expression levels were normalized to that of glyceraldehyde 3-phosphate dehydrogenase (51332, CST) or $\alpha$-tubulin (11224-1-AP, Proteintech, USA). Appropriate secondary antibodies were used to bind the primary antibodies for $1 \mathrm{~h}$. The secondary antibodies used were as follows: anti-rabbit IgG-HRP (7074, CST) and anti-mouse IgG-HRP (7076, CST). Bands were visualized by Immobilon Western Chemiluminescent HRP Substrate (WBKLS0500, Millipore) and Exposure machine (ImageQuant LAS500). ImageJ was used to quantify the bands.

\section{Statistical analysis}

SPSS 22.0 statistical software was used for statistical analysis, and GraphPad Prism 5 was used to prepare graphs. All data are expressed as mean \pm SEM. Data were initially tested for normality and equal variance by Shapiro-Wilk and equal variance test, respectively. Comparisons between two groups were conducted using an $t$ test. To compare the statistical significance of differences among three or more groups, group comparisons were performed using parametric (one-way analysis of variance) and nonparametric (Mann-Whitney $U$ ) tests when normality and equal variance tests failed. Where variances are homogeneous, the least significant difference test is used, while the Tamhane test is employed when variances cannot be considered homogeneous. For all analysis, statistical significance was reported as follows: "P $<0.05 ;{ }^{* * *} P<0.01 ;{ }^{* * *} P<0.001$; n.s., no significance.

\section{Results}

\section{ZDF rats develop cardiac hypertrophy and exhibit impaired cardiac function}

To investigate cardiac hypertrophy in diabetic rats, we first explored the blood glucose levels and body weights of rats at various time points. Blood glucose was significantly increased in ZDF rats from week 9 compared with ZL rats (Fig. $1 \mathrm{~A}, P<0.001$ ). At the same time, ZDF rats gained significantly more body weight compared with ZL rats at week 12 (Fig. $1 \mathrm{~B}, P<0.01$ ). These results indicated that a diabetic rat model was successfully generated. The HW/ tibia length ratio increased significantly in ZDF compared to $\mathrm{ZL}$ rats (Fig. $1 \mathrm{C}, P<0.05$ ). WGA staining showed increased cardiomyocyte size in ZDF rats (Fig. 1D, $P<$ 0.001). Further, the protein levels of hypertrophic markers, including ANP and $\beta-M H C$, were significantly higher in ZDF rat hearts than that in ZL rat hearts (Fig. $1 \mathrm{E}, P<0.01)$. These results imply that ZDF rats develop cardiac hypertrophy. Moreover, echocardiographic assessment of cardiac function showed that LV enddiastolic anterior wall thickness, LV wall end diastole, left ventricular ejection fraction, and left ventricular fractional shortening were increased in ZDF rats compared to ZL rats (Fig. 1 F, $P<0.01$ ). These results indicated that cardiac hypertrophy and compensatory enhancement of systolic function occurred in ZDF rats.

\section{Expression levels of mitochondrial dynamics and calcium handling pathway-related proteins in a hypertrophic hearts of ZDF rats}

To investigate the changes in mitochondrial dynamics induced by hyperglycemia in the hearts of ZDF rats, we measured the protein levels of fission and fusion proteins in myocardial tissue. Mitochondrial fission is mainly regulated by cytoplasmic Drp1, and Drp1 is activated by phosphorylation at S616 and dephosphorylation at S637 (ref. ${ }^{14}$ ). We found that hyperglycemia increased Drp1 phosphorylation at S616, but reduced phosphorylation at S637 (Fig. 2A, B, $P<0.05$ ). The protein expression levels of fusion proteins Opa1 and Mfn2 were significantly decreased in the ZDF group (Fig. $2 \mathrm{C}, P<0.01$ ). To further investigate the effect of the calcium-related signaling pathway on hyperglycemia-induced cardiac hypertrophy, the protein expression levels of Orail, CnA, and p-ERK1/ 2 were examined in myocardial tissue. Interestingly, Orai1, CnA, and p-ERK1/2 protein levels were increased significantly in the ZDF group (Fig. $2 \mathrm{D}-\mathrm{F}, P<0.05$ ). In summary, hyperglycemia increased Orai1, CnA, and pERK1/2 protein levels and fission protein Drp1 activity, but decreased fusion protein levels in the hearts of ZDF rats.

\section{HG induces cardiomyocyte hypertrophy with abnormalities in mitochondrial dynamics and calcium handling}

To examine whether HG induces cardiomyocyte hypertrophy, we treated primary cultured NRCMs with $33 \mathrm{mM}$ glucose (HG) for $72 \mathrm{~h}$. As shown in Fig. 3A, the NRCM area detected by WGA staining was significantly increased in the HG group $(P<0.001)$. Furthermore, protein expression of the cardiac hypertrophic markers $\beta$-MHC and ANP in NRCMs increased significantly in the HG group (Fig. 3B, $P<0.01$ ), indicating that $\mathrm{HG}$ induced NRCM hypertrophy.

To study mitochondrial functional and morphological changes in NRCMs caused by HG, we determined mitochondrial membrane potential (MMP) and mitochondrial 


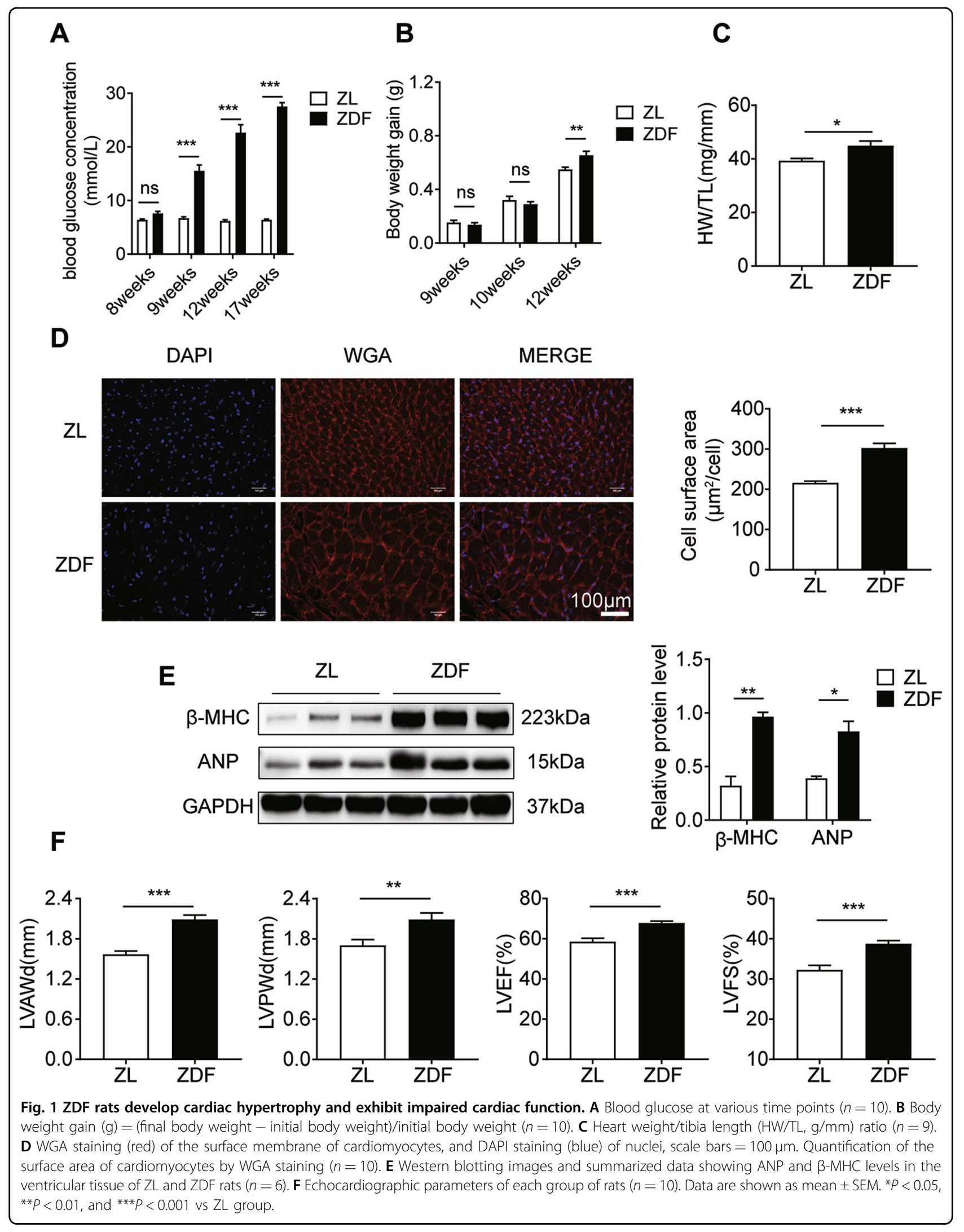




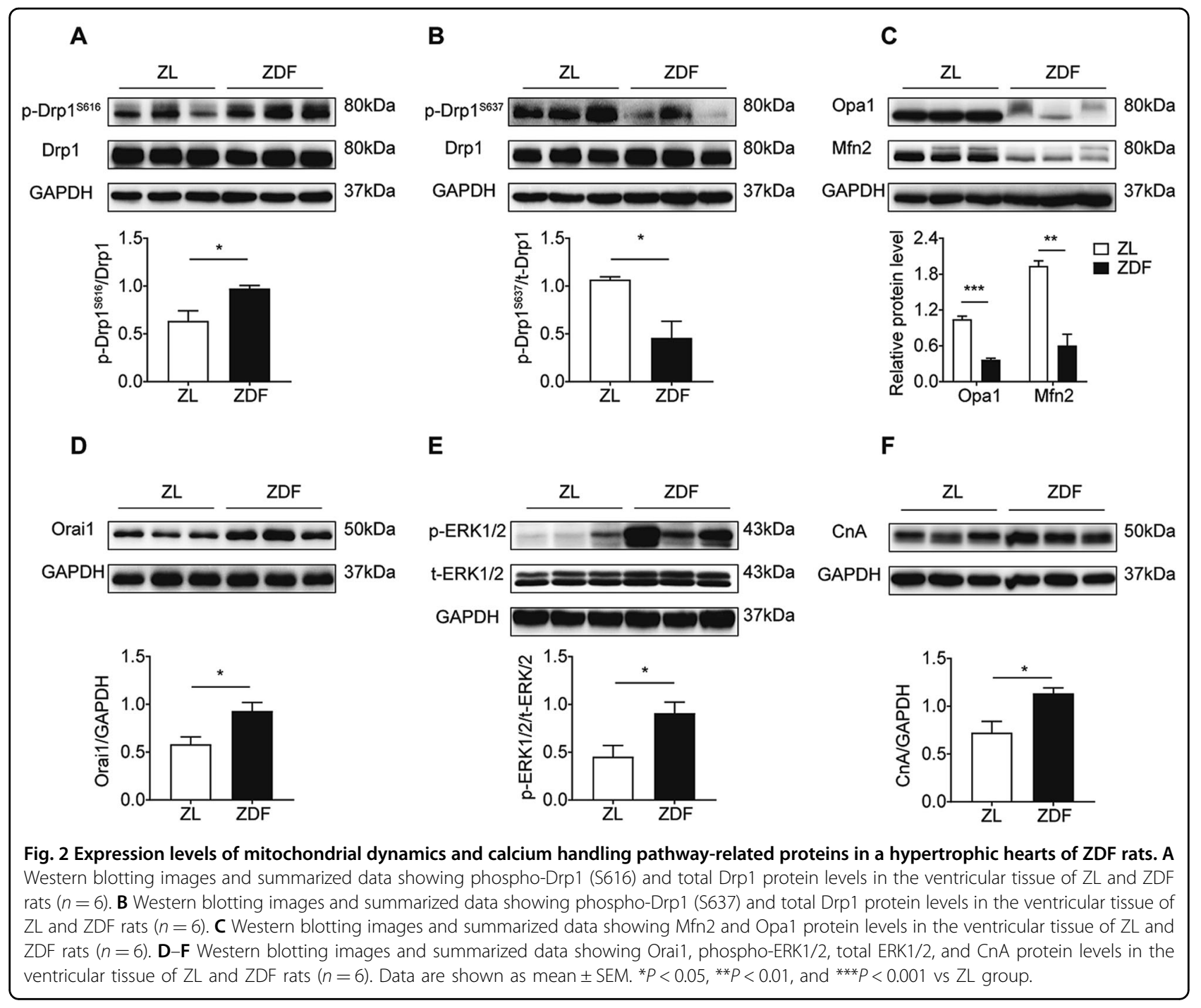

network parameters in NRCMs. In the HG group, NRCM mitochondria tended to be fragmented (decreased number of individuals, networks, and mitochondrial footprint) and showed decreased MMP (Fig. 3C, D, $P<0.01$ ). Furthermore, we found that HG increased the phosphorylation of Drp1 at S616, but decreased phosphorylation at S637 (Fig. 3E, $P<0.01)$. Protein expression levels of the fusion protein Opa1 and Mfn2 were significantly decreased in the HG group (Fig. 3F, $P<0.05$ ). In summary, HG increased Drp1-dependent mitochondrial fission and decreased mitochondrial fusion, resulting in mitochondrial dysfunction and morphological fragmentation.

To further identify the effect of HG on Orail expression and channel function, we also determined the protein expression of Orail in NRCMs. We found that the expression of Orai1 was significantly greater in the HG group compared with the control group (Fig. 3G, $P<0.01$ ). We also determined Orail-mediated $\mathrm{Ca}^{2+}$ entry using the
Fluo4/AM dye in NRCMs. As shown in Fig. 3H, the L-type $\mathrm{Ca}^{2+}$ channel was blocked with $1 \mu \mathrm{M}$ nifedipine. Subsequent addition of $2 \mathrm{mM}$ calcium $10 \mathrm{~min}$ after the addition of $2 \mu \mathrm{M}$ thapsigargin resulted in a larger and sustained increase in $\mathrm{Ca}^{2+}$ entry through the Orai1 channel. Stronger $\mathrm{Ca}^{2+}$ fluorescence was observed in the HG group compared to that in the control groups following stimulation with $\mathrm{CaCl}_{2}(P<0.001)$. There was no statistical difference between the control and HM groups $(P>0.05)$. Accordingly, we confirmed that HG can induce Orai1-mediated intracellular $\mathrm{Ca}^{2+}$ overload by upregulating Orai1.

Mdivi-1 alleviates cardiomyocyte hypertrophy induced by HG by decreasing Drp1-dependent mitochondrial fission

The Mdivi-1 is a selective inhibitor of Drp1, and has been reported to affect ischemia/reperfusion injury and cardiac hypertrophy in animal models ${ }^{15}$. We treated NRCMs with Mdivi-1 to determine whether it could block 


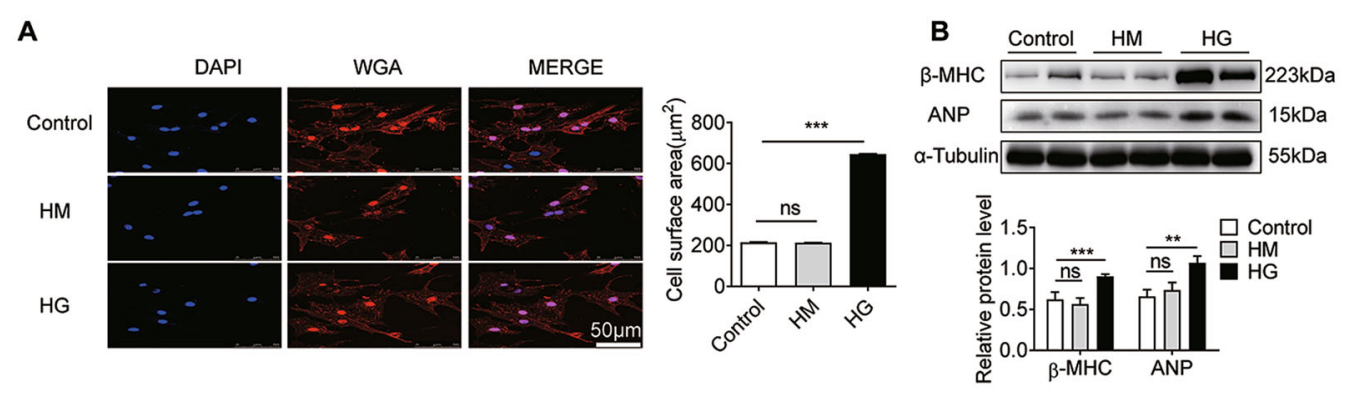

C

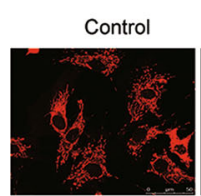

HM

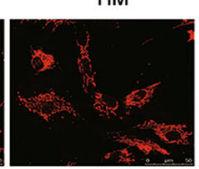

HG
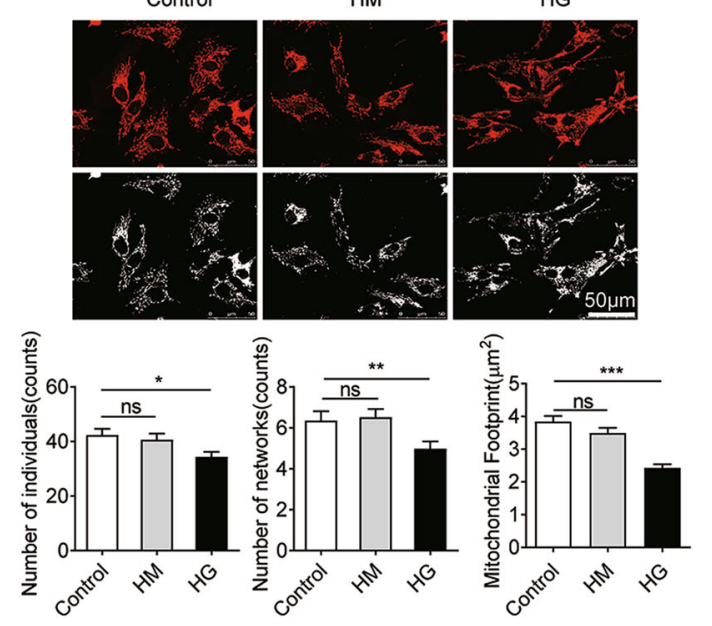

E
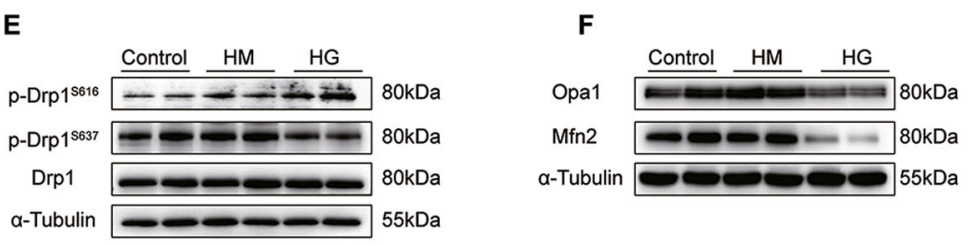

D

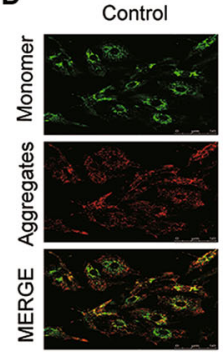

HM

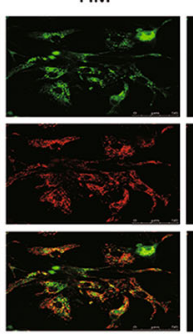

HG
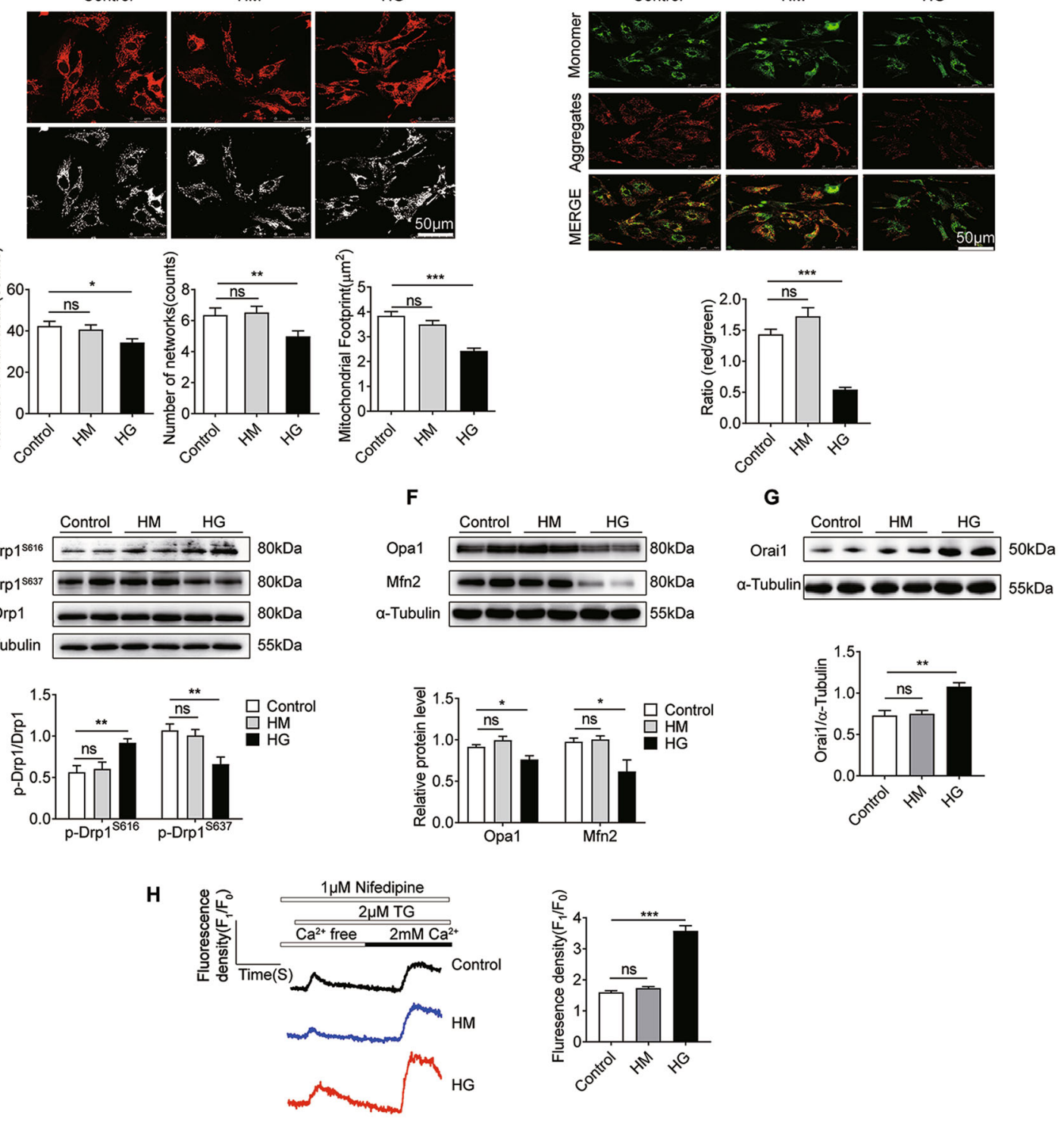

Fig. 3 (See legend on next page.)

cardiomyocyte hypertrophy induced by HG. To this end, the cell surface area and the protein expression levels of $\beta$-MHC and ANP were assessed in NRCMs. As shown in
Fig. 4A, Mdivi-1 significantly inhibited the increased cardiomyocyte area induced by HG $(P<0.001)$. Meanwhile, the upregulation of ANP in NRCMs induced by HG 
(see figure on previous page)

Fig. 3 HG induces cardiomyocyte hypertrophy with abnormalities in mitochondrial dynamics and calcium handling. A WGA staining (red) of the surface membrane of cardiomyocytes, and DAPI staining (blue) of nuclei. Scale bars $=50 \mu \mathrm{m}$. To quantify cardiomyocyte size, HG induced an increase in cardiomyocyte size ( $n=45$ cells). B Western blotting images and summarized data showing $\beta$-MHC $(n=5)$ and ANP $(n=11)$ levels in NRCMs. C Confocal images of mitochondrial morphology in NRCMs. RGB color, scale bars $=50 \mu \mathrm{m}$; 8-bit color, scale bars $=3 \mu \mathrm{m}$. There was a main effect of $\mathrm{HG}$ on the number of individuals and networks, and the size of the mitochondrial footprint. Overall, HG decreased all mitochondrial network parameters $(n=140$ cells). D JC-1 staining of NRCMs. Red fluorescence is from JC-1 aggregates in healthy mitochondria with polarized inner mitochondrial membranes, while green fluorescence is emitted by cytosolic JC-1 monomers and indicates MMP dissipation. Merged images indicate the co-localization of JC-1 aggregates and monomers. Scale bar $=50 \mu \mathrm{m}$. MMP of cardiomyocytes for each group was calculated as the fluorescence ratio of red to green. HG decreased the MMP ( $n=390$ cells). E Western blotting images and summarized data showing Drp1 protein level and phosphorylation at $\mathbf{S 6 1 6}(n=6)$ or $\mathbf{S 6 3 7}(n=8)$ in NRCMs. F Western blotting images and summarized data showing Opa1 $(n=4)$ and Mfn2 $(n=3)$ protein levels in NRCMs. G Western blotting images and summarized data showing Orai1 protein levels in NRCMs $(n=4)$. $\mathbf{H}$ Representative traces of $\mathrm{Ca}^{2+}$ influx in NRCMs are shown. Fluorescence intensity measurements of Fluo4-AM revealed the intracellular $\mathrm{Ca}^{2+}$ concentration in NRCMs following $\mathrm{CaCl}_{2}$ stimulation $\left(n=76\right.$ cells). $F_{1}$ fluorescence intensity, $F_{0}$ baseline fluorescence. Data are shown as mean $\pm \mathrm{SEM}$. ${ }^{*} P<0.05,{ }^{* *} P<0.01$, and ${ }^{* * *} P<0.001 ;$ n.s. no significant statistical difference.

was significantly inhibited by $10 \mu \mathrm{M}$ Mdivi-1 (Fig. 4B, $P<0.01)$. Furthermore, Mdivi-1 significantly reduced the upregulation of $\beta-\mathrm{MHC}$ and ANP induced by HG (Fig. $4 \mathrm{C}, P<0.01)$. These results suggest that Mdivi-1 could alleviate HG-induced cardiac hypertrophy.

Next, we explored the cellular mechanisms by which Mdivi-1 protects against HG-induced cardiomyocyte hypertrophy. As shown in Fig. 4D, mitochondrial fission in Mdivi-1-treated cells was decreased compared with the HG group $(P<0.01)$. Furthermore, the decrease in MMP in HG conditions was dramatically inhibited by Mdivi-1 compared with the HG group (Fig. 4E, $P<0.001$ ). These results indicated that Mdivi-1 improved HG-induced mitochondrial dysfunction.

We then explored the expression of phospho-Drp1 (S616 and S637) and total Drp1 protein levels in NRCMs. As shown in Fig. 4F, Mdivi-1 significantly inhibited the upregulation of $\mathrm{p}$-Drp1 ${ }^{\mathrm{S} 616}$ and downregulation of $\mathrm{p}$ Drp1 ${ }^{\mathrm{S} 637}$ induced by HG $(P<0.01)$. Total Drp1 showed no significant changes. No obvious difference was observed between the control, HM, dimethyl sulfoxide, and Mdivi-1 groups $(P>0.05)$. Collectively, these results indicated that Mdivi-1 inhibited Drp1 phosphorylation at S616 and increased Drp1 phosphorylation at S637, preventing mitochondrial fission induced by HG.

To further test this in vivo, we explored the therapeutic role of Mdivi-1 on DCM. We first examined the effect of Mdivi-1 injection on cardiac hypertrophy in diabetic mice. We then investigated the effect of Mdivi-1 on mitochondrial morphology and ATP level in $\mathrm{db} / \mathrm{db}$ diabetic mice. As shown in Fig. S1A, protein levels of $\beta$-MHC and ANP were increased in $\mathrm{db} / \mathrm{db}$ mice hearts compared with $\mathrm{db} / \mathrm{m}$ mice, but decreased in $\mathrm{db} / \mathrm{db}$ mice after Mdivi-1 treatment. Consistent with in vitro results, $\mathrm{p}$-Drp1 ${ }^{\mathrm{S} 616}$ expression was increased and $\mathrm{p}$-Drp $1^{\mathrm{S} 637}$ expression was decreased in diabetic mice, and Mdivi-1 intervention in vivo reversed these changes (Fig. S1B). In addition, direct ATP measurement revealed that the myocardial
ATP concentration was significantly reduced in $\mathrm{db} / \mathrm{db}$ mice, and a significant increase in $\mathrm{db} / \mathrm{db}+$ Mdivi-1 mice was observed (Fig. S1C, $P<0.05$ ).

The electron microscopy showed that the area and aspect ratio of mitochondria were significantly decreased in $\mathrm{db} / \mathrm{db}$ compared with $\mathrm{db} / \mathrm{m}$ group, which indicated that mitochondrial fission was broadly raised. This alteration was improved by Mdivi-1 injection (Fig. 4G, $P<0.001$ ). No obvious difference was observed between the $\mathrm{db} / \mathrm{m}$ and $\mathrm{db} / \mathrm{db}+$ Mdivi-1 groups $(P>0.05)$.

\section{Orai1 inhibition prevents HG-induced cardiomyocyte hypertrophy and mitochondrial dysfunction in NRCMs}

We previously showed that HG induced Orai1mediated intracellular $\mathrm{Ca}^{2+}$ overload by upregulating Orail expression. Next, we investigated whether inhibition of Orail prevents HG-induced cardiomyocyte hypertrophy in NRCMs. We used BTP2 to block the Orail channel, which is a $\mathrm{Ca}^{2+}$ release-activated $\mathrm{Ca}^{2+}$ channel inhibitor. We found that BTP2 significantly reduced the upregulation of $\beta-\mathrm{MHC}$ and ANP induced by HG (Fig. 5A, $P<0.01$ ). These results suggest that BTP2 could alleviate HG-induced cardiac hypertrophy. Further, the Cas9/sgRNA technique was used to knock down Orai1 in cardiomyocytes. As shown in Fig. 5B, the protein expression levels of Orail in the sgOrail group were significantly decreased compared with the $\operatorname{Neg}$ group $(P<$ $0.01)$. Meanwhile, compared to the HG group, Orai1 protein expression was obviously decreased in the HG + sgOrail group $(P<0.001)$. Furthermore, Orai1 knockdown inhibited HG-induced $\beta$-MHC and ANP expression (Fig. 5C, $P<0.01$ ). According to these results, we concluded that high expression of Orail might be a promoter in cardiomyocyte hypertrophy induced by HG.

We also studied the effects of Orail inhibition on mitochondrial dysfunction induced by HG in NRCMs. As shown in Fig. 5D, much shorter and smaller mitochondria were prevalent in the HG group compared with the control 


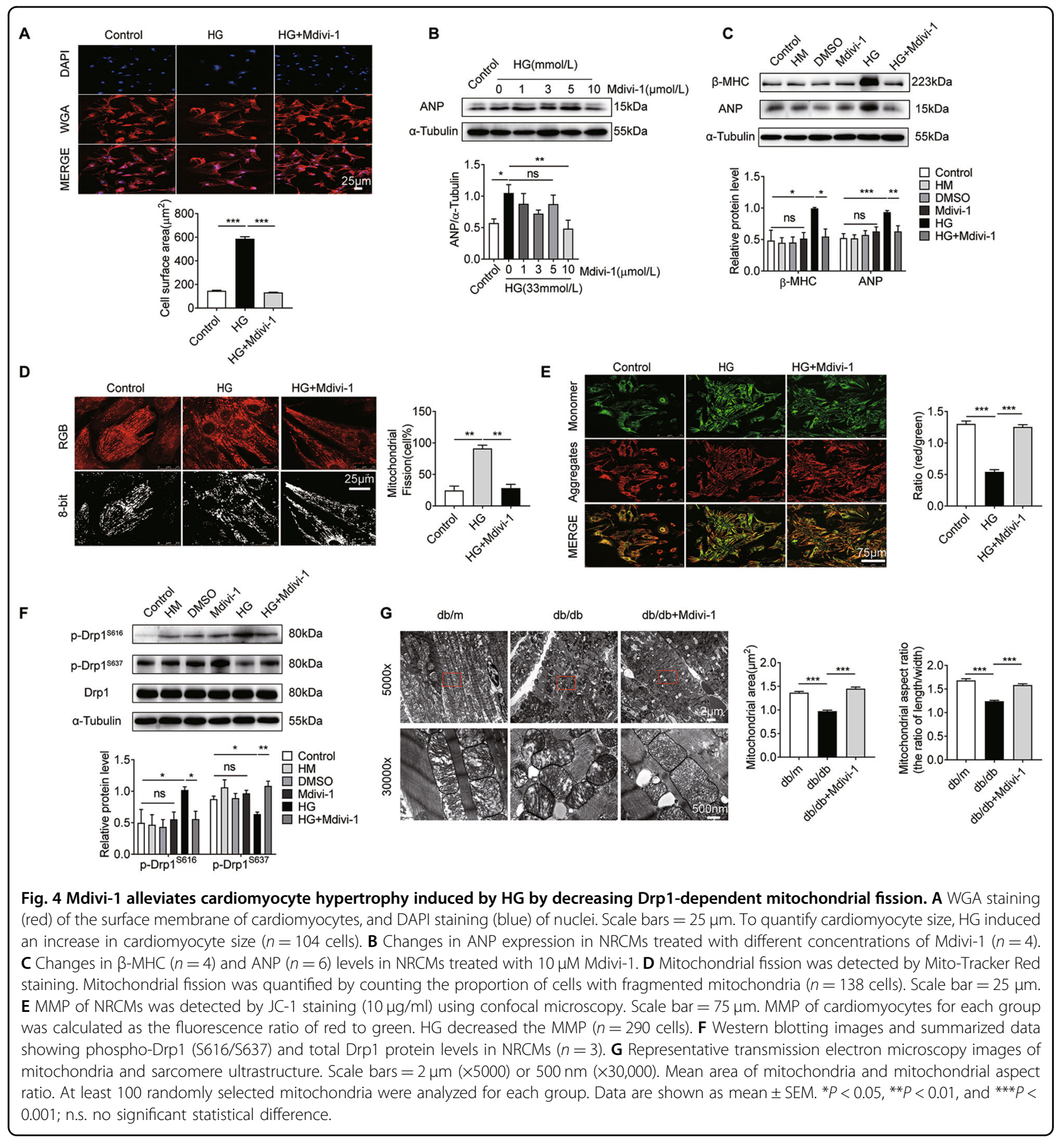

group, indicating mitochondrial fragmentation. Quantitative results showed that mitochondrial fission in BTP2-treated cells was decreased compared with the HG group $(P<0.01)$. Furthermore, the decrease in MMP in the HG condition was dramatically inhibited by BTP2 compared with the HG group (Fig. 5E, $P<0.001$ ). These results indicated that BTP2 improved HG-induced mitochondrial dysfunction.
Orai1 might contribute to HG-induced cardiomyocyte hypertrophy via activation of CnA, ERK-Drp1 pathways

To understand the cellular mechanisms by which Orail-mediated $\mathrm{Ca}^{2+}$ entry contributes to HG-induced cardiomyocyte hypertrophy, we aimed to determine whether CnA and ERK-Drp1 pathways were involved in this process. Therefore, western blotting was used to study the effects of Orai1 inhibitor BTP2 $(10 \mu \mathrm{M})$ 


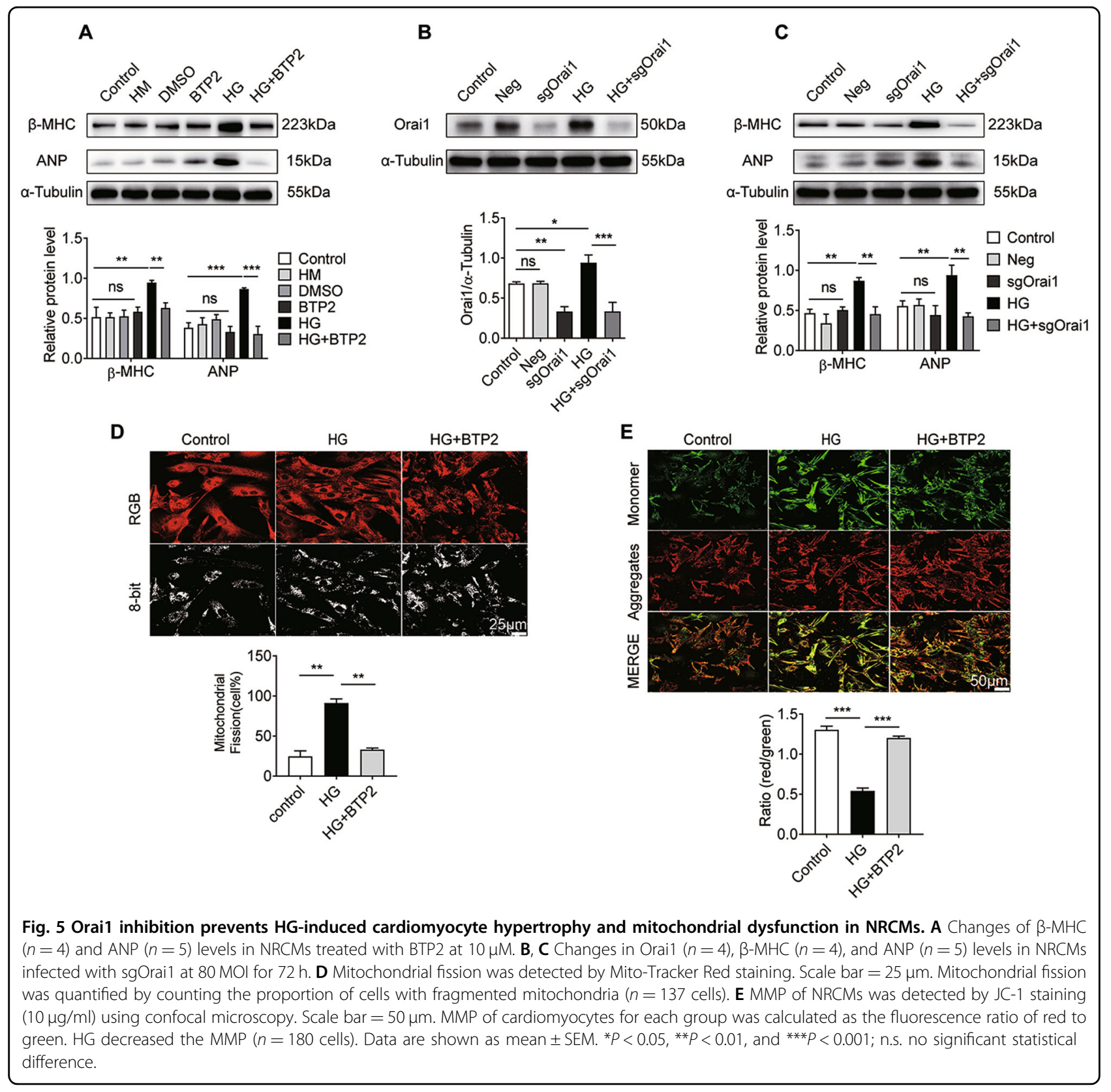

treatment on CnA and the phosphorylation of ERK and Drp1 in NRCMs. We found that BTP2 significantly reduced the upregulation of $\mathrm{CnA}$ in hypertrophic cells induced by HG (Fig. 6A, $P<0.05$ ). BTP2 also significantly inhibited the upregulation of p-ERK1/2 induced by HG $(P<0.05)$. Total ERK1/2 showed no significant changes (Fig. 6B, $P>0.05$ ). Furthermore, BTP2 significantly inhibited the upregulation of $\mathrm{p}-\mathrm{Drp}^{\mathrm{S} 616}$ and downregulation of $\mathrm{p}$-Drp1 ${ }^{\mathrm{S} 637}$ induced by $\mathrm{HG}$ (Fig. $6 \mathrm{C}, P<$ 0.01 ). Orail knockdown also significantly inhibited the upregulation of $\mathrm{p}-\mathrm{Drp1}^{\mathrm{S} 616}$ and downregulation of p-Drp1 ${ }^{\mathrm{S} 637}$ induced by HG (Fig. $6 \mathrm{D}, P<0.01$ ). Total Drp1 showed no significant changes $(P>0.05)$. No obvious difference was observed between the control, Neg, and sgOrail groups $(P>0.05)$. These data demonstrated that CnA, ERK1/2, and Drp1 may be downstream pathways of Orai1-mediated $\mathrm{Ca}^{2+}$ influx; however, the role of $\mathrm{CnA}$ and ERK1/2 in regulating Drp1 phosphorylation in HG-induced cardiomyocyte hypertrophy remains unclear.

\section{CnA and p-ERK1/2 contribute to HG-induced cardiomyocyte hypertrophy by regulating Drp1 phosphorylation}

To clarify the role of CnA and p-ERK1/2 in regulating Drp1 phosphorylation in HG-induced cardiomyocyte 

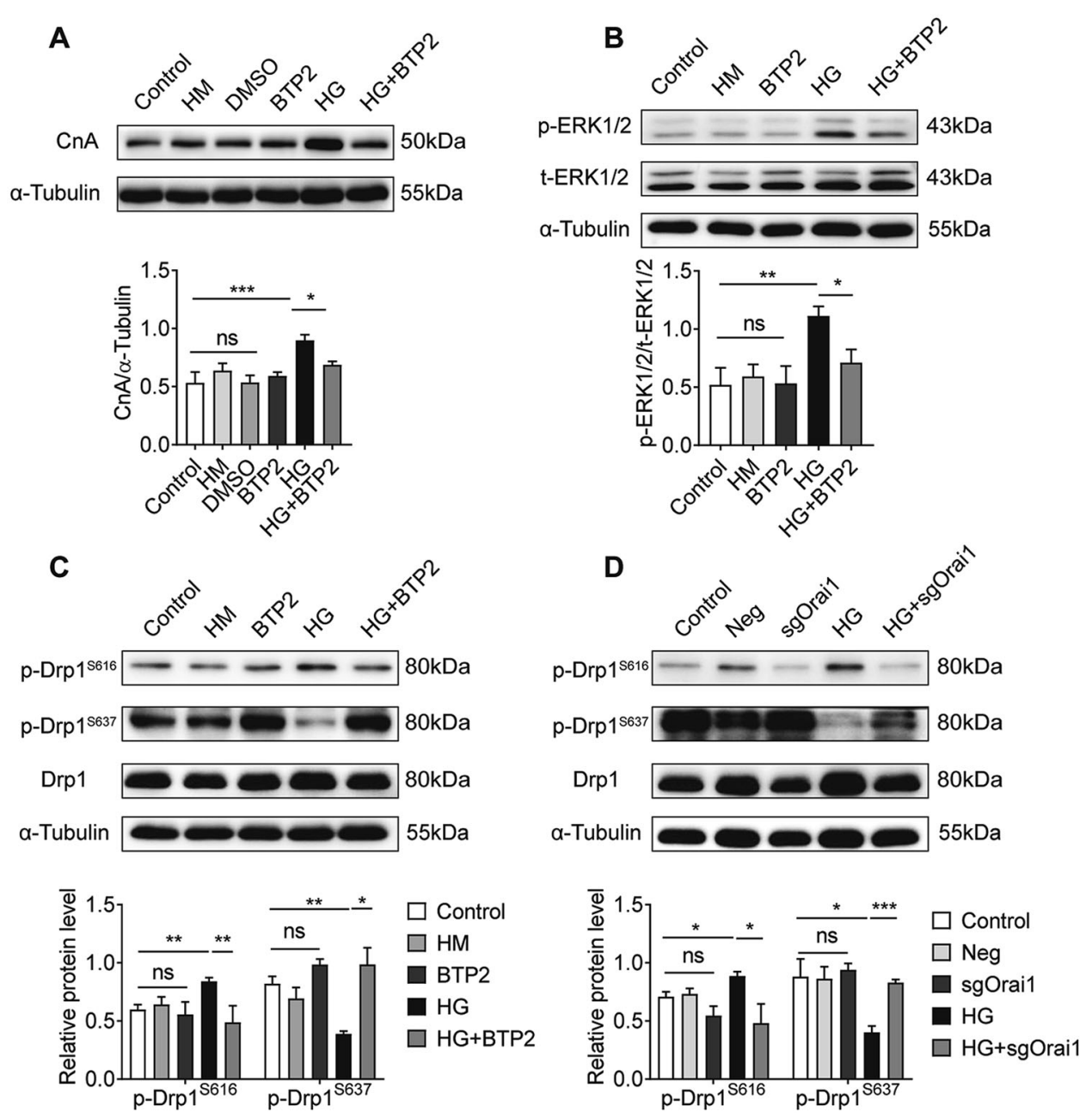

Fig. 6 Orai1 might contribute to HG-induced cardiomyocyte hypertrophy via activation of CnA, ERK-Drp1 pathways. A Changes in CnA levels in NRCMs treated with $10 \mu \mathrm{M} \mathrm{BTP2}(n=4)$. B Changes of p-ERK1/2 and total ERK1/2 protein levels in NRCMs treated with BTP2 $10 \mu M(n=3)$. C Western blotting images and summarized data showing p-Drp1 ${ }^{\mathrm{S616}}(n=4)$ and p-Drp1 ${ }^{\mathrm{S637}}(n=3)$ protein levels in NRCMs treated with $10 \mu \mathrm{M}$ BTP2. D Changes of $\mathrm{p}-\operatorname{Drpl}^{\mathrm{S616}}(n=4)$ and $\mathrm{p}-\mathrm{Drp}^{\mathrm{S637}}(n=4)$ levels in NRCMs infected with sgOrai1 at $80 \mathrm{MOl}$ for $72 \mathrm{~h}$. The protein levels of phospho-Drp1 (S616/S637) were normalized by total Drp1. Data are shown as mean \pm SEM. ${ }^{*} P<0.05,{ }^{* *} P<0.01$, and ${ }^{* * *} P<0.001$; n.s: no significant statistical difference.

hypertrophy, we treated NRCMs with the CnA inhibitor CsA or the p-ERK1/2 inhibitor U0126, respectively. As shown in Fig. 7A, following the addition of $10 \mu \mathrm{M}$ U0126, $\mathrm{p}$-ERK1/2 levels in NRCMs were significantly reduced compared with HG-treated samples $(P<0.01)$. Total ERK1/2 showed no significant changes $(P>0.05)$. We found that U0126 could significantly inhibit the upregulation of ANP induced by HG (Fig. 7B, $P<0.001$ ). Meanwhile, UO126 significantly inhibited the phosphorylation of Drp1 at S616 in a HG environment (Fig. 7C, $P<$ 0.01). Notably, HG increased the abundance of $\mathrm{CnA}$, but the expression of $\mathrm{CnA}$ was not influenced by CsA treatment (Fig. 7D, $P<0.01$ ). Similarly, the expression of ANP in NRCMs treated with CsA was significantly reduced compared with the HG group (Fig. 7E, $P<0.01$ ). CsA significantly induced phosphorylation of Drp1 at S637 in a HG environment (Fig. 7F, $P<0.01$ ). Overall, these data show that mitochondrial fission induced by phosphorylation of Drp1 at S616 or dephosphorylation at S637 is a critical event in HG-induced cardiomyocyte hypertrophy by $\mathrm{p}$-ERK1/2 or CnA activation.

\section{Discussion}

Advanced DCM characterized by LV hypertrophy and hyperglycemia is one of the main causes of metabolic changes in diabetes ${ }^{1}$. Therefore, we cultured NRCMs in HG medium to establish a cell model for studying the pathogenesis of DCM. We report a novel mechanism that 


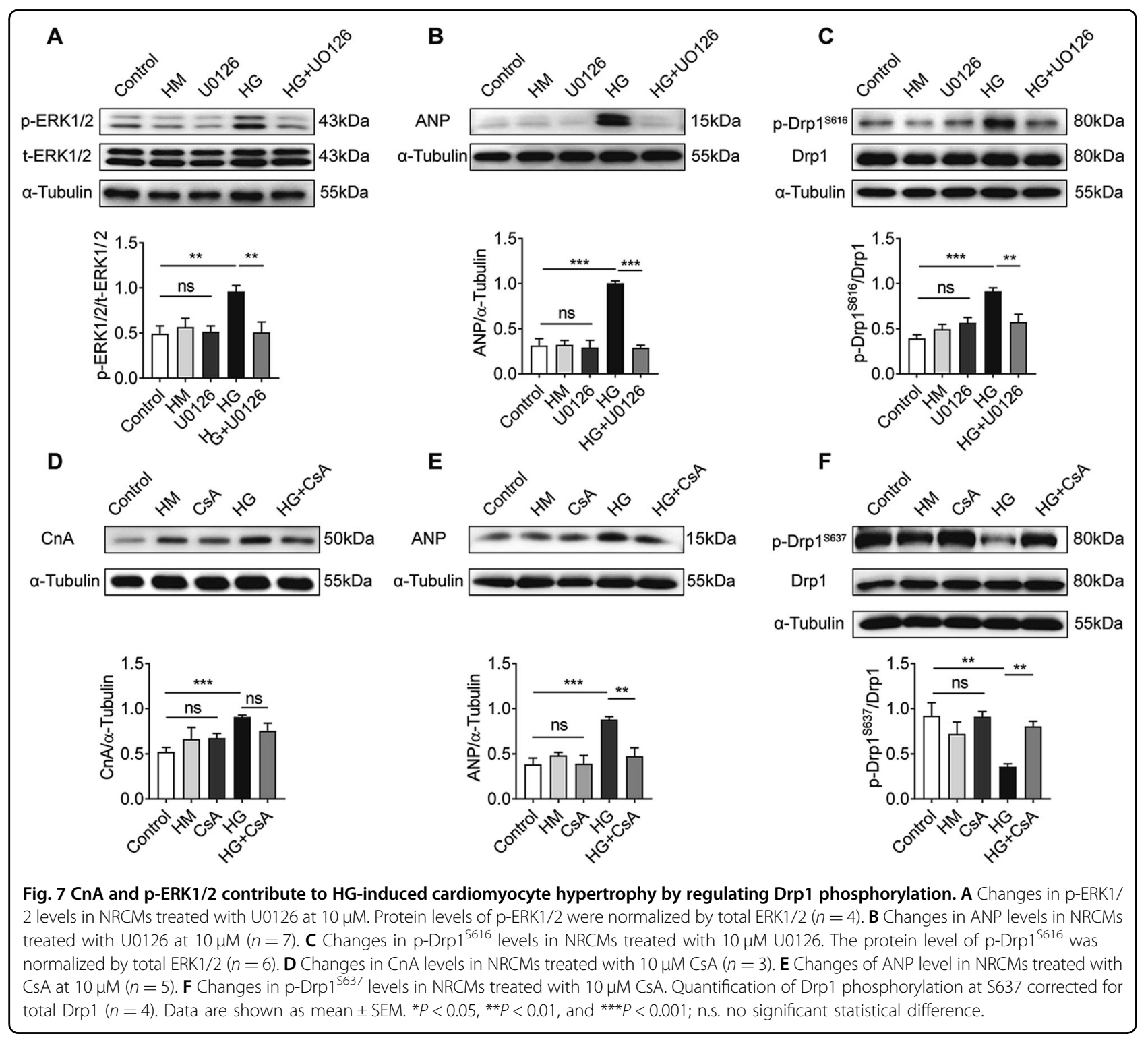

contributes to HG-induced cardiac hypertrophy (Fig. 8). We found that HG increased Orail, CnA, and p-ERK1/2 protein levels and Drp1 activity in vivo and in vitro. Importantly, we showed for the first time that inhibition of Orail-mediated $\mathrm{Ca}^{2+}$ entry reduces HG-induced cardiomyocyte hypertrophy by decreasing Drp1-dependent mitochondrial fission, and that Drp1 activity is regulated by $\mathrm{CnA}$ and p-ERK1/2. This study provides new insights into the role and mechanism of Orail in DCM via mitochondrial dysfunction.

ZDF rats are generally considered a well-characterized model of type 2 diabetes with significant cardiovascular dysfunction. In the present study, as expected, the cardiac function of ZDF rats was impaired. The ratio of HW/TL and the expression level of hypertrophy-related proteins in ZDF rats were significantly increased, indicating that the cardiac dysfunction in diabetic rats primarily manifested as cardiac hypertrophy. Notably, we found that Drp1 and Orail signaling pathway-related proteins were activated in hypertrophic rat hearts. However, the detailed mechanism of the Drp1 and Orail signaling pathway in DCM remains obscure.

The role of mitochondrial dynamics-related proteins in cardiomyopathy has been studied ${ }^{16}$. The main mitochondrial fusion protein Mfn2 was downregulated in cardiac hypertrophy. Opa1 protein was significantly decreased after heart failure and myocardial infarction. Drp1 contributes to the promotion of hypertensive cardiac hypertrophy ${ }^{17}$. Drp1 activity is activated by phosphorylation at S616 and dephosphorylation at S637 (ref. ${ }^{18}$ ). Moreover, previous reports have shown that inhibition of Drp1-mediated mitochondrial fission could reduce mitochondrial dysfunction and cardiac 


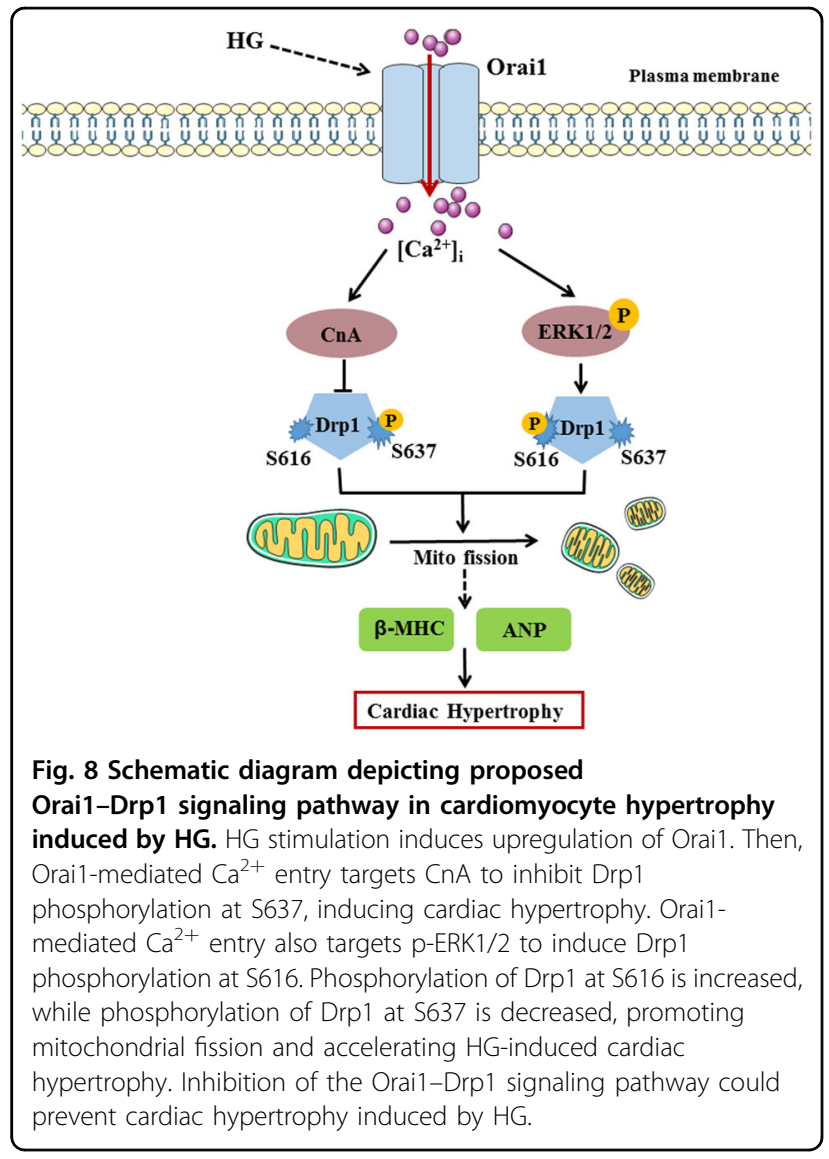

dysfunction in diabetic mice ${ }^{19}$. Notably, the effect of phosphorylation at different sites of Drp1 on DCM is unknown. In the present study, we showed that HG induces cardiomyocyte hypertrophy with abnormalities in mitochondrial dynamics. HG promoted Drp1-dependent mitochondrial fission and decreased mitochondrial fusion, leading to mitochondrial dysfunction. Thus, we considered that inhibition of Drp1 could attenuate the cardiomyocyte hypertrophy induced by HG. Mdivi- 1 is a cell-permeable selective inhibitor of Drp1-mediated mitochondrial fission. A previous study indicated that Mdivi-1 improves mitochondrial function and cardiac function after myocardial infarction in diabetic mice ${ }^{20}$. However, the mechanisms through which this compound functions directly in DCM remain unknown. Importantly, we found that Mdivi-1 could inhibit HGinduced cardiomyocyte hypertrophy by decreasing Drp1 phosphorylation at S616 or increasing phosphorylation at S637 in NRCMs. In addition, treatment with Mdivi-1 restores mitochondrial morphology and ATP production along with the inhibition of cardiac hypertrophy in the diabetic mice. It turns out that Drp1-mediated mitochondrial fission promotes diabetic cardiac hypertrophy by decreasing myocardial energy storage. However, the underlying mechanism by which HG regulates Drp1 phosphorylation remains unclear.
A previous study showed that disorders of mitochondrial dynamics are related to abnormal calcium levels ${ }^{21}$. We have shown that HG induced expression of Orail and Orai1mediated $\mathrm{Ca}^{2+}$ entry. But it is unclear whether Orai1mediated $\mathrm{Ca}^{2+}$ entry is associated with mitochondrial dysfunction in DCM. Previous studies showed that inhibition of the upregulation of stromal interaction molecule 1/Orai1 prevents cardiac hypertrophy ${ }^{22}$ and that SOCE plays an important role in activating transcriptional pathways associated with cardiomyocyte hypertrophy ${ }^{23}$. Our study demonstrated that inhibition of Orai1-mediated SOCE by BTP2 prevents cardiomyocyte hypertrophy and mitochondrial dysfunction induced by HG. In addition, knockdown of Orail attenuated HG-induced cardiomyocyte hypertrophy. Together, these studies indicate the involvement of Orail-mediated $\mathrm{Ca}^{2+}$ entry in HG-induced cardiomyocyte hypertrophy and mitochondrial dysfunction.

Furthermore, we found that BTP2 significantly inhibited the upregulation of $\mathrm{CnA}$ and p-ERK1/2 in NRCMs treated with HG. Consistent with previous results, Orai1 knockdown may play a role in myocardial hypertrophy by reducing $\mathrm{CnA}$ and ERK1/2 activity $^{24-26}$. Restoring intracellular $\mathrm{Ca}^{2+}$, downregulating the expression of $\mathrm{CnA}$, inhibiting the phosphorylation of Drp1 at S616, and increasing the phosphorylation of Drp1 at S637 could prevent mitochondrial fission in cardiomyocytes ${ }^{27}$. Consistent with previous results, this study showed that both BTP2 treatment and Orai1 knockdown significantly inhibited the increase in $\mathrm{p}-\mathrm{Drp} 1^{\mathrm{S} 616}$ expression and decrease in p-Drp1 ${ }^{\mathrm{S} 637}$ expression induced by HG. Next, we verified that Orail may be involved in the regulation of Drp1 activity through CnA or ERK pathways in DCM.

Previous studies have shown that inhibiting the levels of $\mathrm{Ca}^{2+}$ in the cytoplasm contributes to reduce mitochondrial fission and improve mitochondrial and heart function ${ }^{28}$. In addition, ERK1/2 promotes Drp1 phosphorylation, leading to mitochondrial dysfunction in heart failure ${ }^{29}$. Studies have been reported that the ERK signaling pathway induces phosphorylation of Drp1 at S616 (ref. ${ }^{30}$ ). Activation of ERK-mediated mitochondrial Drp1 phosphorylation at S616 promotes chemotherapy resistance in colorectal cancer $^{31}$ and inhibition of p-ERK1/2 by U0126 suppresses mitochondrial fission by decreasing Drp1 phosphorylation at S616 in Huntington's disease mutant cells ${ }^{32}$. Consistent with previous results, we found that Drp1 phosphorylation at S616 is increased in hypertrophic cardiomyocytes induced by HG; this effect was abolished by treatment with U0126. In addition, previous studies have shown that the $\mathrm{Ca}^{2+}$-dependent phosphatase calcineurin regulates translocation of Drp1 to mitochondria through dephosphorylation of $\mathrm{S} 637$ in HeLa cells ${ }^{6}$. Most functional studies of calcineurin have been performed with its inhibitor CsA (ref. ${ }^{33}$ ). This study found that CsA could significantly inhibit HG-induced cardiomyocyte hypertrophy 
by increasing mitochondrial fission via promotion of dephosphorylation of Drp1 at S637.

In summary, we believe that HG could induce intracellular $\mathrm{Ca}^{2+}$ overload by upregulating Orai1. Orai1-mediated $\mathrm{Ca}^{2+}$ influx activates ERK or CnA-Drp1 pathway, leading to diabetic cardiac hypertrophy via promoting mitochondrial fission. Inhibition of Orail- $\mathrm{Ca}^{2+}{ }_{-} \mathrm{CnA}$ or ERK-Drp1 signaling pathways could prevent cardiomyocyte hypertrophy induced by HG. Our results suggest that targeting Orai1-Drp1 axis may offer a promising approach to ameliorate the cardiac hypertrophy associated with type $2 \mathrm{DM}$.

\section{Acknowledgements}

We are grateful to Fei-Hong Lian, Li Zhang, Meng-Yuan Zhou, Peng Zeng, HaiDan Liang, Xiao-Yue Qin, Shu-Zhen Chen, and Sui-Min Li (South China University of Technology, Guangzhou, China) for participating in the experiment and writing the manuscript.

\begin{abstract}
Author details
${ }^{1}$ Guangdong Provincial Key Laboratory of Clinical Pharmacology, Research Center of Medical Sciences, Guangdong Provincial People's Hospital, Guangdong Academy of Medical Sciences, 510080 Guangzhou, Guangdong, China. ${ }^{2}$ Guangdong Cardiovascular Institute, Guangdong Provincial People's Hospital, Guangdong Academy of Medical Sciences, 510080 Guangzhou, Guangdong, China. ${ }^{3}$ School of Medicine, South China University of Technology, 510006 Guangzhou, Guangdong, China. ${ }^{4}$ School of Biological Science and Engineering, South China University of Technology, 510006 Guangzhou, Guangdong, China. ${ }^{5}$ School of Pharmaceutical Sciences, Southern Medical University, 510515 Guangzhou, Guangdong, China. ${ }^{6}$ Department of Nephrology, Guangdong Provincial People's Hospital, Guangdong Academy of Medical Sciences, 510080 Guangzhou, Guangdong, China
\end{abstract}

\section{Author contributions}

C.-Y.D. and C.-B.C. conceived and designed the study. Q.-R.W., D.-L.Z., and P.-M.L. collected the data, analyzed the data, and wrote the manuscript. H.Y., L.-A.L., S.-J.K., Y.-Y.L., F.R., Y.-M.X., J.-J.L., and S.-X.L. assisted with the data collection and participated in the writing of the manuscript. All authors read and approved the final manuscript.

\section{Funding}

This work was supported by grants from the Natural Science Foundation of Guangdong Province (2019A1515011933, 2017B030311010), High-level Hospital Construction Plan (DFJH201925, DFJH201808, DFJH201901), and National Key Research and Development Project (2018YFC1312502).

\section{Conflict of interest}

The authors declare no competing interests.

\section{Publisher's note}

Springer Nature remains neutral with regard to jurisdictional claims in published maps and institutional affiliations.

Supplementary information The online version contains supplementary material available at https://doi.org/10.1038/s41419-021-03502-4.

Received: 27 August 2020 Revised: 2 February 2021 Accepted: 4 February 2021

Published online: 26 February 2021

\section{References}

1. Parim, B., Sathibabu Uddandrao, V. V. \& Saravanan, G. Diabetic cardiomyopathy: molecular mechanisms, detrimental effects of conventional treatment, and beneficial effects of natural therapy. Heart Fail. Rev. 24, 279-299 (2019).
2. Jia, G., Hill, M. A. \& Sowers, J. R. Diabetic cardiomyopathy: an update of mechanisms contributing to this clinical entity. Circ. Res. 122, 624-638 (2018).

3. Galloway, C. A. \& Yoon, Y. Mitochondrial dynamics in diabetic cardiomyopathy. Antioxid. Redox Signal. 22, 1545-1562 (2015).

4. Yan, K., Wang, K. \& Li, P. The role of post-translational modifications in cardiac hypertrophy. J. Cell. Mol. Med. 23, 3795-3807 (2019).

5. Kashatus, J. A. et al. Erk2 phosphorylation of Drp1 promotes mitochondrial fission and MAPK-driven tumor growth. Mol. Cell 57, 537-551 (2015).

6. Cereghetti, G. M. et al. Dephosphorylation by calcineurin regulates translocation of Drp1 to mitochondria. Proc. Natl Acad. Sci. USA 105, 15803-15808 (2008).

7. Zheng, S., Leclerc, G. M., Li, B., Swords, R. T. \& Barredo, J. C. Inhibition of the NEDD8 conjugation pathway induces calcium-dependent compensatory activation of the pro-survival MEK/ERK pathway in acute lymphoblastic leukemia. Oncotarget 9, 5529-5544 (2017).

8. Dewenter, M., von der Lieth, A., Katus, H. A. \& Backs, J. Calcium signaling and transcriptional regulation in cardiomyocytes. Circ. Res. 121, 1000-1020 (2017).

9. Rusnak, F. \& Mertz, P. Calcineurin: form and function. Physiol. Rev. 80, 1483-1521 (2000)

10. Prakriya, M. \& Lewis, R. S. Store-operated calcium channels. Physiol. Rev. 95 1383-1436 (2015).

11. Luo, R., Gomez, A.-M., Benitah, J.-P. \& Sabourin, J. Targeting Orai1-mediated store-operated $\mathrm{Ca}(2+)$ entry in heart failure. Front. Cell Dev. Biol. 8, 586109-586109 (2020).

12. Bartoli, F. et al. Orai1 channel inhibition preserves left ventricular systolic function and normal $\mathrm{Ca}(2+)$ handling after pressure overload. Circulation 141, 199-216 (2020).

13. Valente, A. J., Maddalena, L. A., Robb, E. L., Moradi, F. \& Stuart, J. A. A simple ImageJ macro tool for analyzing mitochondrial network morphology in mammalian cell culture. Acta Histochem. 119, 315-326 (2017).

14. Pagliuso, A., Cossart, P. \& Stavru, F. The ever-growing complexity of the mitochondrial fission machinery. Cell. Mol. Life Sci. 75, 355-374 (2018).

15. Hasan, P. et al. Mitochondrial fission protein, dynamin-related protein 1, contributes to the promotion of hypertensive cardiac hypertrophy and fibrosis in Dahl-salt sensitive rats. J. Mol. Cell. Cardiol. 121, 103-106 (2018).

16. Dorn, G. II Mitochondrial fission/fusion and cardiomyopathy. Curr. Opin. Genet. Dev. 38, 38-44 (2016).

17. Hasan, P. et al. Mitochondrial fission protein, dynamin-related protein 1, contributes to the promotion of hypertensive cardiac hypertrophy and fibrosis in Dahl-salt sensitive rats. J. Mol. Cell. Cardiol. 121, 103-106 (2018).

18. Chang, C.-R. \& Blackstone, C. Dynamic regulation of mitochondrial fission through modification of the dynamin-related protein Drp1. Ann. NY Acad. Sci. 1201, 34-39 (2010)

19. Ding, M. et al. Melatonin prevents Drp1-mediated mitochondrial fission in diabetic hearts through SIRT1-PGC1 a pathway. J. Pineal Res. 65, e12491 (2018).

20. Ding, $M$. et al. Inhibition of dynamin-related protein 1 protects against myocardial ischemia-reperfusion injury in diabetic mice. Cardiovasc. Diabetol. 16, 19 (2017).

21. Shanmughapriya, $\mathrm{S}$. et al. $\mathrm{Ca}^{2+}$ signals regulate mitochondrial metabolism by stimulating CREB-mediated expression of the mitochondrial $\mathrm{Ca}^{2+}$ uniporter gene MCU. Sci. Signal. 8, ra23 (2015).

22. Dai, F. et al. Overexpression of SARAF ameliorates pressure overload-induced cardiac hypertrophy through suppressing STIM1-Orai1 in mice. Cell. Physiol. Biochem. 47, 817-826 (2018).

23. Prakriya, M. \& Lewis, R. S. Store-operated calcium channels. Physiol. Rev. 95, 1383-1436 (2015)

24. Voelkers, M. et al. Orai1 and Stim1 regulate normal and hypertrophic growth in cardiomyocytes. J. Mol. Cell. Cardiol. 48, 1329-1334 (2010).

25. Wang, L.et al. Orai1 mediates tumor-promoting store-operated $\mathrm{Ca}(2+)$ entry in human gastrointestinal stromal tumors via C-KIT and the extracellular signal-regulated kinase pathway. Tumour Biol. 39, https://doi.org/ 10.1177/1010428317691426 (2017).

26. Yu, B.-X. et al. Inhibition of Orai1-mediated $\mathrm{Ca}(2+)$ entry limits endothelial cell inflammation by suppressing calcineurin-NFATc4 signaling pathway. Biochem. Biophys. Res. Commun. 495, 1864-1870 (2018).

27. Yang, Y. et al. Extract of Sheng-Mai-San ameliorates myocardial ischemiainduced heart failure by modulating $\mathrm{Ca}(2+)$-calcineurin-mediated Drp1 signaling pathways. Int. J. Mol. Sci. 18, 1825 (2017).

28. Wu, S. N. et al. Binding of FUN14 domain containing 1 with inositol 1,4,5trisphosphate receptor in mitochondria-associated endoplasmic reticulum 
membranes maintains mitochondrial dynamics and function in hearts in vivo. Circulation 136, 2248-224 (2017).

29. Huang, C.-Y. et al. Inhibition of ERK-Drp1 signaling and mitochondria fragmentation alleviates IGF-IIR-induced mitochondria dysfunction during heart failure. J. Mol. Cell. Cardiol. 122, 58-68 (2018).

30. Cai, J. et al. ERK/Drp1-dependent mitochondrial fission is involved in the MSCinduced drug resistance of T-cell acute lymphoblastic leukemia cells. Cell Death Dis. 7, e2459-e2459 (2016).
31. Huang, C. et al. HMGB1 promotes ERK-mediated mitochondrial Drp1 phosphorylation chemoresistance RAGE colorectal cancer. Cell Death Dis. 9, 1004 (2018).

32. Roe, A. J. \& Qi, X. Drp1 phosphorylation by MAPK1 causes mitochondrial dysfunction in cell culture model of Huntington's disease. Biochem. Biophys. Res. Commun. 496, 706-711 (2018).

33. Molkentin, J. D. Parsing good versus bad signaling pathways in the heart: role of calcineurin-nuclear factor of activated T-cells. Circ. Res. 113, 16-19 (2013). 